\title{
Cost Comparison of 2 Video Laryngoscopes in a Large Academic Center
}

\author{
Adam Thaler, DO, Dalmar Mohamod, MD, Andrew Toron, BS, and Marc C. Torjman, PhD
}

Objective: Retrospective study examining hospital cost information of patients requiring endotracheal intubation with video laryngoscopy. Provide a practical cost assessment on use of the McGRATH and GlideScope video laryngoscopes (VLS).

Methods: This study examined 52 hospital locations within a single, large university hospital, with most of those locations being hospital operating rooms. A total of 34600 endotracheal intubations performed over 24 months, of which 11345 were video laryngoscopies. Electronic medical records containing demographic data and information related to endotracheal intubation procedures, with monthly breakdowns between GlideScope and McGRATH intubations, were reviewed. Cost information calculated for equipment, blades, batteries, repairs, and subsequent analysis performed to determine cost differences between those 2 instruments during the COVID-19 period.

Results: A total of 5501 video laryngoscopy procedures were performed using the McGRATH VL and 5305 were performed using the GlideScope VL. Costs over 24 months were $\$ 181093$ lower (55.5\%) for McGRATH compared to GlideScope. The mean (SD) monthly costs for GlideScope blades were $\$ 3837$ ( $\$ 1050$ ) and $\$ 3236$ (\$538) for years 1 and 2, respectively, vs $\$ 1652$ (\$663) and $\$ 2933$ (\$585) for McGRATH blades $(P<.001)$. Most total cost differences were attributed to equipment and blade purchases, which were $\$ 202595$ (65.0\%) higher for GlideScope. During the COVID-19 period, the use of the McGRATH increased to $61 \%$ of all video laryngoscopy cases, compared to $37 \%$ for GlideScope $(P<.001)$. Blade cost difference for the COVID19 period was $\$ 128$ higher for the McGRATH even though 293 more intubations were performed with that device.

Conclusions: Use of the McGRATH resulted in a cost savings of $55 \%$ compared to the GlideScope, and its use was highest during the COVID-19 period, which may be explained by its more portable and practical features.

Keywords: video laryngoscope; McGRATH; GlideScope; endotracheal intubation; hospital costs; COVID-19.
$\mathrm{H}$ ospitals have come to rely on video laryngoscopes (VLs) for tracheal intubation as necessary tools for better visualization of airways. Modern video laryngoscopy developed in the $2000 s^{1}$ as a progression from direct laryngoscopy, which began in 1852 when Horace Green used a bent tongue spatula and sunlight to examine a child. ${ }^{2}$ VLs have seen many improvements and adaptations of their own, resulting in many different styles and types circulating around hospitals. The GlideScope (Verathon Inc, Bothell, WA) and the McGRATH (Medtronic, Minneapolis, MN) are examples of such instruments, which are now widely used in the US and are the 2 VLs of choice at our institution.

A few studies have compared VLs to direct laryngoscopes. In their systematic review, Lewis et al have shown the numerous benefits of using a VL over a direct laryngoscope. Some general conclusions were that the use of video laryngoscopy reduced the number of failed intubations, decreased laryngeal trauma, and provided improved visualizations. ${ }^{3}$ Other studies have compared the different types of VLs, including the McGRATH and the GlideScope, examining factors such as intubation time and display quality of the image. Two studies found that medical students were equally successful at using both the McGRATH and the GlideScope, ${ }^{4,5}$

From the Department of Anesthesiology, Thomas Jefferson University and Hospitals, Sidney Kimmel Medical College, Philadelphia, PA, and Sidney Kimmel Medical College at Thomas Jefferson University, Philadelphia, PA. 
while another study found that care providers using the GlideScope had quicker intubation times. ${ }^{6}$ Lastly, Savoldelli et al concluded that more providers preferred the McGRATH, which provided better laryngeal views, ${ }^{7}$ while their subsequent study showed more favorable learning curves of the Airtraq compared to the McGRATH and other VLs. ${ }^{8}$

Although there have been no reported differences in safety and effectiveness of the McGRATH and GlideScope devices, cost data on the use of these 2 popular laryngoscopes are lacking. Such information is important considering the increasing costs of medical technologies and the significant financial losses experienced by health care systems due to the COVID-19 crisis. The purpose of this retrospective cohort study was to compare the cost efficiency of the McGRATH MAC and GlideScope Core VLs at a large academic center.

\section{Methods}

This retrospective study was performed under exemption from the Thomas Jefferson University Institutional Review Board. The primary data sources consisted of hospital electronic patient records (EPIC) and cost information from the device manufacturers and hospital staff. The electronic patient data were provided by the EPIC Enterprise Analytics Business Intelligence group at Thomas Jefferson University Hospital (Center City Campus, Philadelphia, PA), while device costs were obtained from Verathon, Medtronic, and departmental staff responsible for purchasing equipment. Monthly data were obtained over a 24-month period (June 2018 through May 2020) when the McGRATH VL was placed into use in the department of anesthesiology. The 2 types of VLs were made available for use in a total of 52 locations, with the majority being hospital operating rooms.

The following variables were recorded: number of endotracheal intubations performed each month with breakdown between video laryngoscopy and flexible bronchoscopy airways, frequency of use for each type of laryngoscope, blades used, and equipment costs for use of each laryngoscope. Hospital cost estimates for both the McGRATH and GlideScope laryngoscopes included batteries, handles, blades, and the devices themselves. Cost data were also collected on frequency of device failure, maintenance, and replacement of parts and lost equipment.

\section{Analysis}

De-identified electronic medical records consisted of nominal and quantitative variables, with demographic data and information related to the endotracheal intubation procedure. All data were in chronological order and sorted by date after which coding was applied, to identify device type and allocate pertinent cost information. Descriptive statistics were reported as mean (SD) and sum for costs; frequency tables were generated for intubation procedures according to device type and time periods. Data were analyzed using the $\chi^{2}$ test, the student $t$ test, and the Wilcoxon Mann-Whitney $U$ test, with a $P$ value set at .05 for statistical significance. SPSS version 26 and GraphPad Prism version 6 were used for all statistical analyses.

\section{Results}

A total of 34600 endotracheal intubations were performed over the 24-month study period, and 11345 (32.8\%) were video laryngoscopy procedures. Out of all video laryngoscopy procedures, 5501 (48.5\%) were performed using the McGRATH VL and 5305 (46.8\%) were conducted using the GlideScope VL. The difference of 539 (4.8\%) cases accounts for flexible bronchoscopy procedures and endotracheal intubations using other video laryngoscopy equipment. The mean (SD) monthly number of video laryngoscopy procedures for the 24 months was 221 (54) and 229 (89) for the GlideScope and McGRATH devices, respectively. Monthly endotracheal intubation distributions over 24 months trended upward for the McGRATH VL and downward for the GlideScope, but there was no statistically significant $(P=.71)$ difference in overall use between the 2 instruments (Figure 1).

To examine the observed usage trends between the $2 \mathrm{VL}$ during the first and last 12 months, a univariate ANOVA was conducted with the 2 time periods entered as predictors in the model. Video laryngoscopy intubations were performed $(P=.001)$ more frequently with the GlideScope during the first 12 months; however, use of the McGRATH VL increased $(P<.001)$ during the following 12 months compared to GlideScope. The GlideScope 


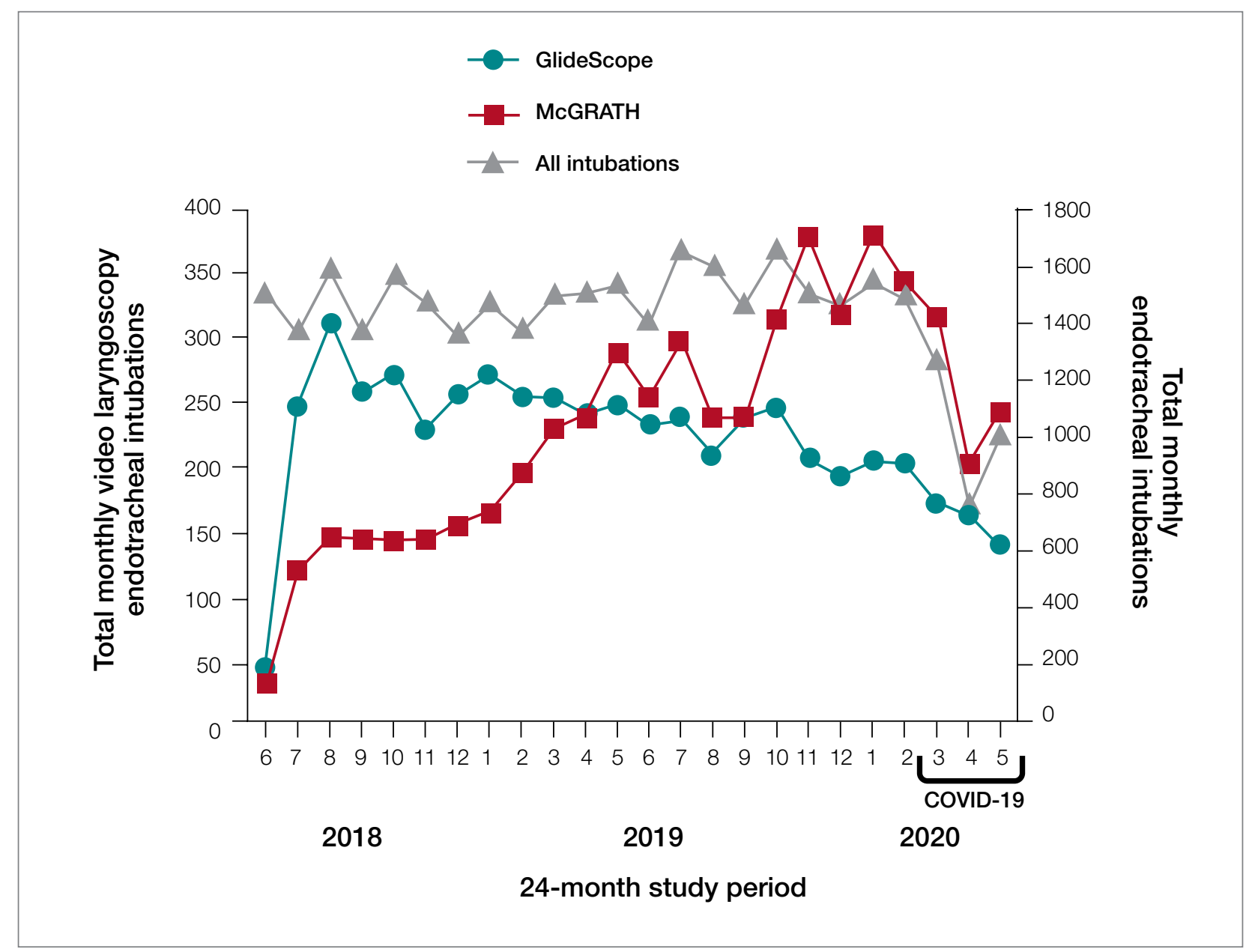

Figure 1. Monthly counts of video laryngoscopy procedures.

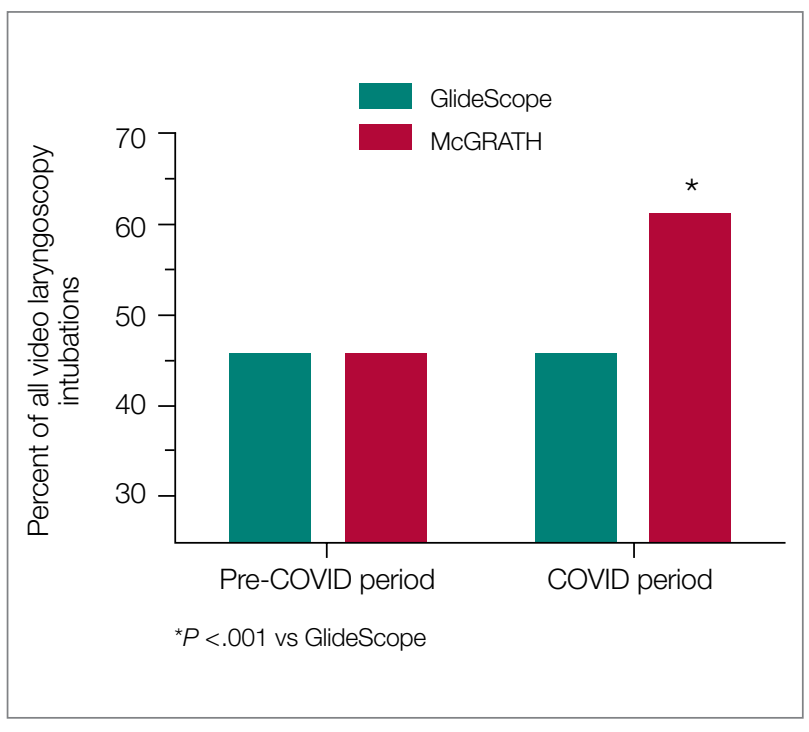

Figure 2. The McGRATH is the more popular device during COVID. accounted for $54 \%$ of all VL intubations during the first 12 months, with the McGRATH accounting for $58 \%$ of all video laryngoscopy procedures for months 12 to 24 . Additionally, the increase in video laryngoscopy procedures with the McGRATH during the last 3 months of the study period was despite an overall reduction in surgical volume due to the COVID-19 crisis, defined for this study as March 1, 2020, to May 31, 2020 (Figure 1). There was a statistically significant $(P<.001)$ difference in the case distribution between use of the McGRATH and GlideScope $V L$ for that period. The anesthesia personnel's use of the McGRATH VL increased to $61 \%$ of all video laryngoscopy cases, compared to 37\% for the GlideScope (Figure 2).

The total costs calculated for equipment, blades, and repairs are presented in Table $\mathbf{1}$ and yearly total costs are shown in Figure 3. Overall costs were $\$ 181093$ 


\begin{tabular}{|c|c|c|c|c|c|}
\hline & $\begin{array}{l}\text { McGRATH units } \\
\text { purchased }\end{array}$ & $\begin{array}{c}\text { McGRATH } \\
\text { costs }\end{array}$ & $\begin{array}{c}\text { GlideScope } \\
\text { units purchased }\end{array}$ & $\begin{array}{l}\text { GlideScope } \\
\text { costs }\end{array}$ & $\begin{array}{c}\text { Difference \$ } \\
(\%)\end{array}$ \\
\hline Equipment & 36 & $\$ 54000$ & 15 & $\$ 226725$ & $\begin{array}{c}172725 \\
(-319.86)\end{array}$ \\
\hline Blades & 5501 & $\$ 55010$ & 5305 & $\$ 84880$ & $\begin{array}{c}29870 \\
(-54.30)\end{array}$ \\
\hline Batteries & 104 & $\$ 3526$ & 0 & No cost & - \\
\hline Repairs/Losses & $15^{a}$ & $\$ 22500$ & 3 & $\$ 14000^{b}$ & $\begin{array}{c}8500 \\
(37.77)\end{array}$ \\
\hline Stylets & 5501 & $\$ 10177$ & 20 & $\$ 700^{c}$ & $\begin{array}{c}9477 \\
(93.12)\end{array}$ \\
\hline Total & - & $\$ 145212$ & - & $\$ 326305$ & $\begin{array}{l}181093 \\
(-55.50)\end{array}$ \\
\hline $\begin{array}{l}\text { a Lost devices } \\
\text { b Baton replacements } \\
{ }^{c} \text { Reusable item }\end{array}$ & & & & & \\
\hline
\end{tabular}

lower (55.5\%) for the McGRATH VL compared to the GlideScope over the 24-month period. The mean (SD) monthly costs for GlideScope VL blades were \$3837 (\$1050) and \$3236 (\$538) for years 1 and 2, respectively, vs $\$ 1652$ (\$663) and $\$ 2933$ (\$585) for the McGRATH VL blades. Most of the total cost differences were attributed to equipment and blade purchases, which were \$202595 (65.0\%) higher for the GlideScope compared to the McGRATH VL. The monthly blade costs alone were higher $(P<.001)$ for the GlideScope over the 2-year period; however, the McGRATH VL required use of disposable stylets at a cost of $\$ 10177$ for all endotracheal intubations, compared to $\$ 700$ for the GlideScope device.

An analysis was performed to determine whether costs differed between those 2 instruments during the COVID-19 period. There was a statistically significant $(P<.001)$ difference in the case distribution between use of the McGRATH and GlideScope VLs during that period. The calculated blade cost difference for the COVID period was $\$ 128$ higher for the McGRATH even though 293 more intubations were performed with that device (Table 2).

\section{Discussion}

We attempted to provide useful cost estimates by presenting pricing data reflecting the approximate cost that

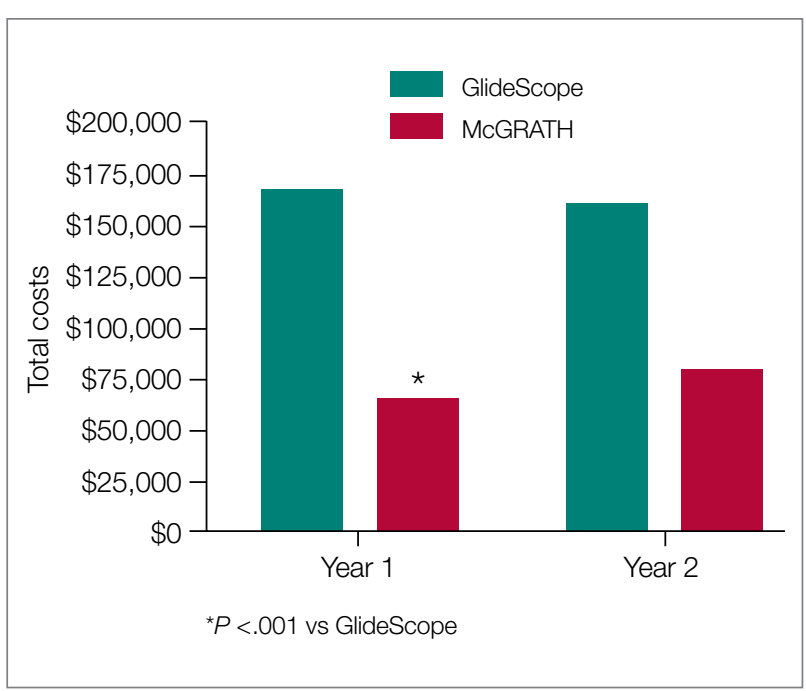

Figure 3. GlideScope costs twice as much as McGRATH.

most large institutional anesthesia practices would incur for using those 2 specific devices and related peripherals. The main findings of our analysis showed that use of the McGRATH MAC VL resulted in a 55\% cost savings compared to the GlideScope, with a similar number of cases performed with each device over the 24-month study period. We believe this represents a substantial savings to the department and institution, which has prompted internal review on the use of video laryngoscopy equipment. None of the McGRATH units failed; however, the GlideScope required 3 baton replacements. 
Table 2. How COVID-19 Affected Case Distribution ${ }^{2}$

\begin{tabular}{lccccc}
\hline & GlideScope & $\begin{array}{c}\text { GlideScope } \\
\text { \% of all VL }\end{array}$ & McGRATH & $\begin{array}{c}\text { McGRATH\% } \\
\text { of all VL }\end{array}$ & P value \\
\hline Pre-COVID period total VL cases & 4838 & 50.5 & 4741 & 49.5 & $<.001^{\text {b }}$ \\
\hline COVID period total VL cases & 467 & 37.5 & 760 & 61.0 & \\
\hline Pre-COVID period blade costs & $\$ 77408$ & 62.0 & $\$ 47410$ & 38.0 & $<.001^{\text {b }}$
\end{tabular}

aThe 21 months defined as the "pre-COVID-19 period" were from June 2018 through February 2020, and the 3 months of the "COVID period" were from March 2020 through May 2020.

'Indicates a statistically significant relationship between the pre-COVID-19 period and the COVID-19 period.

VL, video laryngoscope.

Of note, use of the McGRATH MAC increased during the COVID-19 period, which may be explained by the fact that the operators found it to be a more portable device. Several physicians in the department commented that its smaller size made the McGRATH MAC more practical during the time when a plexiglass box was being used around the patient's head to shield the intubator from aerosolized viral particles.

Although this study demonstrated the cost-saving value of the McGRATH over the GlideScope, a suggested next step would be to examine resource utilization related to video laryngoscopy use. The more dynamic tracking of the use of these devices should facilitate the assessment of existing related resources and decision making, to optimize the benefits of this initiative. We would anticipate reduced use of anesthesia personnel, such as technicians to assist with the management of this device which could be significant. As new respiratory viruses are appearing each year, video laryngoscopy will continue to gain increasing use in operating rooms and acute care locations. The adding of protective barriers between patients and providers calls for use of the most practical and effective $V L$ devices, to protect personnel who are at high risk of contamination from airway secretions and aerosolized particles. ${ }^{9,10}$

The COVID-19 pandemic has demonstrated the value of anesthesiology in regards to analyzing and finding solutions to effectively manage infected patients or those suspected of infection in the perioperative environment. Inexpensive products are often avoided because cheaper devices are associated with being of lower quality. However, the association with cost and quality-and the assumption that a higher price is positively correlated with higher quality-is overall inconsistent in the medical literature. ${ }^{11} \mathrm{~A}$ more effective or higher quality treatment does not necessarily cost more and may actually end up costing less, ${ }^{12}$ as was the case in this study. We have been able to directly cut departmental expenses by using a more efficient and cost-effective device for intubations, without compromising safety and efficacy. Future studies should determine whether this significant reduction in costs from video laryngoscopy intubations with the McGRATH VL will be sustained across anesthesiology departments in the Jefferson Health Enterprise Hospitals, or other health systems, as well as its impact on workflow and personnel resources.

This analysis was restricted to one of the campuses of the Jefferson Health Enterprise. However, this is the largest anesthesia practice, encompassing several locations, which should reflect the general practice patterns across other anesthesiology departments in this large institution. The costs for the devices and peripherals may vary across anesthesia practices depending on volume and contracts negotiated with the suppliers. It was not possible to estimate this variability, which could change the total costs by a few percentage points. We recognize that there may be other costs associated with securing the McGRATH VL to prevent loss from theft or misplacement, which were not included in the study. Lastly, the inability to obtain randomized samples for the 2 groups treated with each device opens up the possibility of selection bias. There were, however, multiple intubators who were free to select 1 of the devices for 
endotracheal intubation, which may have reduced the effect of selection bias.

\section{Conclusion}

This study demonstrated that over a 24-month period use of the McGRATH MAC VL resulted in a cost reduction of around 55\% compared to using the GlideScope for endotracheal intubation procedures performed at a major academic center. Over the first 3 months of the COVID-19 crisis, which our study included, use of the McGRATH VL increased while GlideScope use decreased. This was most likely related to the portability and smaller size of the McGRATH, which better facilitated intubations of COVID19 patients.

Acknowledgements: The authors thank Craig Smith, Senior Anesthesia Technician, for his assistance with the cost information and excellent record-keeping related to the use of video laryngoscopes.

Corresponding author: Marc C. Torjman, PhD, Professor, Department of Anesthesiology, Sidney Kimmel Medical College at Thomas Jefferson University, 111 South 11th St, Suite G-8290, Philadelphia, PA 19107; Marc.Torjman@Jefferson.edu.

Financial disclosures: Dr. Thaler has served as a consultant for Medtronic since September 2020. He has participated in 2 webinars on the routine use of video laryngoscopy.

Funding: This study was supported by the Department of Anesthesiology at Thomas Jefferson University.

doi:10.12788/jcom.0055

\section{References}

1. Channa AB. Video laryngoscopes. Saudi J Anaesth. 2011; 5(4):357-359.

2. Pieters BM, Eindhoven GB, Acott C, Van Zundert AAJ. Pioneers of laryngoscopy: indirect, direct and video laryngoscopy. Anaesth Intensive Care. 2015;43(suppl):4-11.

3. Lewis SR, Butler AR, Parker J, et al. Videolaryngoscopy versus direct laryngoscopy for adult patients requiring tracheal intubation. Cochrane Database Syst Rev. 2016;11(11):CD011136.

4. Kim W, Choi HJ, Lim T, Kang BS. Can the new McGrath laryngoscope rival the GlideScope Ranger portable video laryngoscope? A randomized manikin study. Am J Emerg Med. 2014;32(10):1225-1229.

5. Kim W, Choi HJ, Lim T, et al. Is McGrath MAC better than Glidescope Ranger for novice providers in the simulated difficult airway? A randomized manikin study. Resuscitation. 2014;85(suppl 1):S32.

6. Jeon WJ, Kim KH, Yeom JH, et al. A comparison of the Glidescope to the McGrath videolaryngoscope in patients. Korean $J$ Anesthesiol. 2011;61(1):19-23.

7. Savoldelli GL, Schiffer E, Abegg C, et al. Comparison of the Glidescope, the McGrath, the Airtraq and the Macintosh laryngoscopes in simulated difficult airways. Anaesthesia. 2008; 63(12):1358-1364.

8. Savoldelli GL, Schiffer E, Abegg C, et al. Learning curves of the Glidescope, the McGrath and the Airtraq laryngoscopes: a manikin study. Eur J Anaesthesiol. 2009;26(7):554-558.

9. Schumacher J, Arlidge J, Dudley D, et al. The impact of respiratory protective equipment on difficult airway management: a randomised, crossover, simulation study. Anaesthesia. 2020;75(10):1301-1306.

10. De Jong A, Pardo E, Rolle A, et al. Airway management for COVID19: a move towards universal videolaryngoscope? Lancet Respir Med. 2020;8(6):555.

11. Hussey PS, Wertheimer S, Mehrotra A. The association between health care quality and cost: a systematic review. Ann Intern Med. 2013;158(1):27-34.

12. Mitton C, Dionne F, Peacock S, Sheps S. Quality and cost in healthcare: a relationship worth examining. Appl Health Econ Health Policy. 2006;5(4):201-208. 